\title{
WIFE: Wireless Indoor Positioning Based on Fingerprint Evaluation ${ }^{\star}$
}

\author{
Apostolia Papapostolou and Hakima Chaouchi \\ Telecom-Sudparis, CNRS SAMOVAR, UMR 5157, LOR department \\ \{apostolia.papapostolou, hakima. chaouchi\}@it-sudparis.eu
}

\begin{abstract}
Location awareness of a user or a device has always been considered as a key element for enhancing network performance and improving user experience by enabling efficient mobility and innovative location-based services. In this paper, we present our proposal named WIFE (Wireless Indoor positioning based on Fingerprint Evaluation), a user-based location determination system which utilizes the information of the Signal Strength (SS) received from the surrounding Access Points (APs) inside a building. We focus on a WiFi environment for its low cost and ease of deployment and study fingerprint-based deterministic techniques for their simplicity and reduced processing time and resource requirements. We first address the inherent impairments of an indoor environment which prevent a positioning system from being accurate and then describe our proposed methodology for mitigating them.
\end{abstract}

Keywords: Wireless networks, Location, Positioning System.

\section{Introduction}

Advances in mobile computing, wireless networking and portable devices have fostered mobility of the users while connected. Information regarding the location of the user at any time is important not only for facilitating network management tasks, like resource management, network planning, load balancing etc. but also for enabling the so called location-based services or more broadly speaking context-aware applications, with location being a key attribute of the term context. The user can interact with his surrounding environment based on this location information. The goal of a position determination scheme is to provide an accurate, real-time and reliable estimation of the user or device current location.

Positioning systems can be classified regarding various parameters: indoor or outdoor, hardware dependent or independent, deterministic or probabilistic based on the technique, WiFi, Bluetooth, infrared, ultrasound, ultra-wideband depending on the technology. In this paper, we focus on WiFi-based indoor positioning systems and study deterministic methods for their simplicity and low

\footnotetext{
* This paper is conducted in the French national research project ANR 2006 SUN:

Situated and Ubiquitous Networks.
} 
complexity. The strength of the signal received at an unknown position from an AP is related to its distance from the AP's position and thus this information can be utilized for estimating the unknown position. The main benefits of employing WiFi technology for positioning are that WLAN infrastructure is already available and the number of WiFi-enabled devices is rapidly increasing in the markets.

On the other hand, using WiFi for localization has shortcomings as well. Since the main target of a WLAN is the communication between its components, the placement and deployment of the APs are such that minimum overlapping is achieved, undesirable for localization purposes. Moreover, the inherent characteristics of the wireless medium, the so called propagation losses, and uncontrollable environmental changes cause undesirable variations of the SS deteriorating the positioning process. These hindrances become more intense in an indoor environment due to the presence of walls, objects and people movements.

In this paper, we first identify how these inherent impairments of the wireless and indoor characteristics cause SS variations and then describe how our proposed system, WIFE, tries to mitigate them. More specifically, we propose orientation-specific SS calibration of the area before the real-time localization process. Also, a simple pre-processing of both the calibrated and real-time SS samples is proposed for testing their quality and reliability before applying them to the position estimation algorithm. Finally, a modified version of the basic position estimation technique is described for improving the performance of the system regarding both the accuracy and time response factors.

The remainder of this paper is organized as follows: in section 2 we refer to work related to localization issues. In section 3 the main hindrances while applying localization methods in an indoor environment are addressed. In section 4 we describe and model the architecture and the positioning methodology followed by our system and in section [5 we some discuss further enhancements that can be applied. Section 6 gives the simulation results for the experimental data we applied to our system. Finally, in section 7 we conclude our work and identify future directions.

\section{Related Work}

The significance of the location information for many applications but also the difficulty in obtaining an accurate and fast location estimation without expensive hardware-dependent solutions have attracted the interest of the research and industry communities for many years in this topic. For indoor positioning the proposed algorithms can be classified in three major classes: triangulation, proximity and scene analysis, 9].

Triangulation can be done via lateration, which uses multiple distance measurements between known points, or via angulation, which measures angle or bearing relative to points with known separation. Lateration-based techniques assume a radio propagation model which is easy to derive for an outdoor environment but almost impossible for a complicated indoor scene. Angulation-based 
approaches, on the other hand, rely on complex and expensive hardware. Another family of localization techniques is known as proximity which measures the nearness to a known set of points. Thus, the estimated location is not very accurate and can be utilized for specific only applications. Finally, scene analysis or fingerprint-based examines a view from particular vantage points and tries to infer the given unknown location based on the similarity between these views. Depending on the selected format for quantifying this view we can further classify this technique in deterministic or probabilistic. Scene analysis schemes are mostly preferred for indoor positioning.

Many proposals can be found in the literature. Some of them, such as [6], [5], 7], are based on specific hardware. Some others, like 8], use sensors to sense environmental changes and adapt to them. WLAN-based systems, more related to our work, include RADAR, 1, HORUS, 3] and COMPASS, 4]. In RADAR a deterministic based approach is followed and a propagation model is also proposed for predicting the SS variations. HORUS is a probabilisticbased approach which tries to exploit the correlation between successive samples for increasing the accuracy of the system. Finally, COMPASS, also based on a probabilistic model, incorporates orientation information for dealing with SS variations due to the human body blocking effects.

\section{Problem Description}

The main idea of a WiFi-based positioning system is that the strength of the signal received from an AP is indicative of the distance from this AP because of its attenuation with the increase in distance. Thus, combining SS levels from multiple APs the location of the receiver can be inferred. The stability of the SS values at a specific point and the uniqueness of these SS values among different points are two desirable factors for inferring accurately a location based on the available SS information. Unfortunately, unpredictable and uncontrollable SS variations due to inherent characteristics of the wireless medium and the indoor environment make positioning inside a building, compared to an outdoor environment, more complicated and fusing. In the following these main impairments are addressed.

\subsection{Orientation Issues}

It has already been identified that the power level of the signal received from an AP at a fixed location depends on the orientation of the user due to the blocking effect of the human body. For instance, consider the case when a user's orientation is such that his WiFi-receiver has direct line of sight with an AP but for the opposite orientation his body fully prevents a signal from being received from the same AP and the same physical position. Thus, the relation between a position and SS level is not unique (1-1), but, in contrast, different SS values correspond to the same position.

In RADAR, the authors try to deal with this by sampling SS for 4 different orientations and taking the maximum value as representative for this position. 
In COMPASS, the authors assume the existence of digital compasses during the calibration and the online phases.

\subsection{Environmental Changes}

The existence of walls, objects, people in an indoor environment and the often rearrangement of these cause propagation losses (reflection, scattering, diffraction) and thus prevent the signal strength from remaining stable. In RADAR, the authors propose a radio propagation model depending on the number of walls and the construction material in order to model and predict the SS variations. However, we believe that it is difficult to identify the appropriate model for each indoor environment and furthermore some environmental changes are completely random and cannot be incorporated in any model. For example, consider the case when one or more persons enter between the user and an AP while the SS sampling task is performed. It is obvious that the strength of the signal will change even though both location and orientation are fixed. In the Horus system, the authors observe high autocorrelation between successive samples and propose a first order autoregressive model in order to account for this high autocorrelation.

\subsection{Aliasing}

Aliasing is a term used to describe the phenomenon when two points even physically far apart are very similar in signal space. This is mainly because of the complex indoor propagation environment. Apparently, since a WiFi-based location system is based on the similarity in SS to infer the location, aliasing should be eliminated. In 2], considering the location history of the tracked user is proposed. Thus, if a point close in signal space is far away from the previous location of the user, it is not considered as a candidate for inferring the current position. Hardware-based solutions can provide the location system with a location approximate and thus points not within this range are excluded.

\section{WIFE: Architecture and Methodology}

This section presents the general architecture and components of the positioning process followed by the WIFE system.

\subsection{Positioning Architecture}

WLAN-based location determination systems can be categorized into two classes from architectural point of view: switch-based and user-based. In the former, a specific positioning component is required which measures the SS of the mobile user devices within its range and estimates their location based on a specific positioning algorithm. In the user-based approach, the user device is responsible for measuring the SS from the visible APs and in the sequence uses this SS 
information and a positioning algorithm to infer its location. We assume WIFE follows a user-based architecture so no special component is required and therefore it can easily work with the available WLAN system. However, the limited resources of user devices impose the requirement for a positioning process with low processing overheads.

\subsection{Positioning Methodology Overview}

Figure 1 illustrates an overview of the positioning methodology followed by the WIFE system. It includes two main phases, namely the offline and online. During the offline phase, orientation-specific calibration of the area under study is conducted. These SS sample measurements are processed by the SS sample filter module, transformed to an appropriate format and finally stored in a database. The final format of the SS samples is called $S S$ fingerprint and the database Radio Map $(R M)$. Note that the offline phase is conducted only once and repeated only in the case of big environmental changes which may affect the SS characteristics of this area. The online phase is actually the main phase of the positioning process. Initially, as in the offline phase, run-time SS sampling and filtering of these samples are performed. The resulting online SS fingerprint is compared with the $R M$ fingerprints and a subset of RM entries is selected according to a mathematical positioning algorithm. Finally, during the location estimation step, the unknown location of the user device is estimated based on the known location of the selected RM entries.

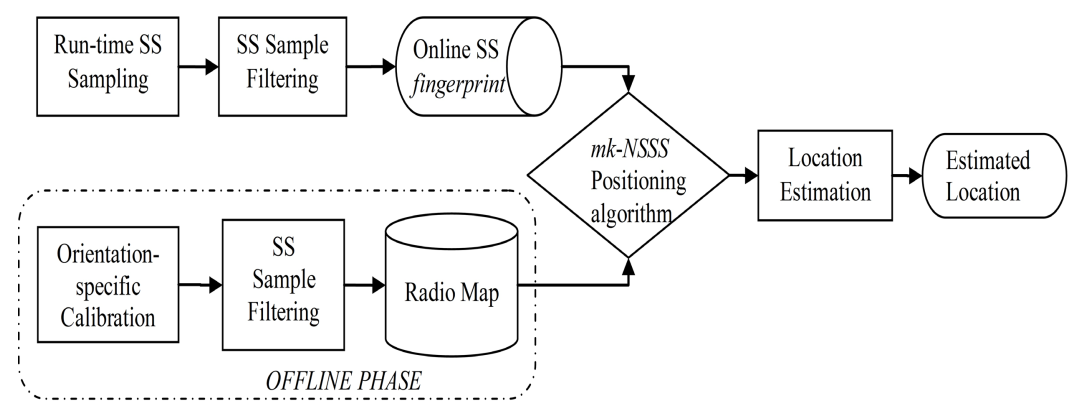

Fig. 1. Positioning Process Overview

In the following the system components are described with more details.

\subsection{Orientation-Specific Calibration and Radio Map}

Since orientation is responsible for SS variations in large extent (see subsection 3.1), we claim that the orientation information should certainly be considered by the positioning system. During the offline phase, we perform an orientationspecific calibration of the area under study. Assume $r$ physically distinct points within the building are selected for training the system and $i: i \in\{1, \ldots, r\}$ one 
of these with known location $\left(x_{i}, y_{i}\right)$. Instead of ignoring the orientation at this point and simply sampling SS measurements from all APs, we propose repeating this SS sampling process for eight possible orientations, i.e. $\forall \omega_{j}$ with $j=1 \ldots 8$. Let $\mathbf{S}_{i}\left(\omega_{j}\right)=\left[S S_{i 1}\left(\omega_{j}\right), S S_{i 2}\left(\omega_{j}\right), \ldots, S S_{i n}\left(\omega_{j}\right)\right]$ the SS sample vector at position $\left(x_{i}, y_{i}\right)$ and orientation $\omega_{j}$, where $S S_{i 1}\left(\omega_{j}\right)$ the SS from $A P_{1}$. This has already been proposed in the COMPASS system where digital compasses are employed for that purpose.

However, we follow a different approach during the Radio Map construction. Let $\left(x_{i}, y_{i}, \mathbf{S}_{i}\right)$ a RM entry, where $\mathbf{S}_{i}$ the SS fingerprint at point $\left(x_{i}, y_{i}\right)$, which should have a format such that the orientation factor is accounted. In COMPASS, the format of $\mathbf{S}_{i}$ depends on the current user orientation, $\omega$, which implies that users need to be equipped with a compass. More precisely, the SS samples of the similar orientations of each calibrated point $\left(x_{i}, y_{i}\right)$ are merged to finally describe the $S S$ fingerprint of the corresponding RM entry $i$, i.e. $\mathbf{S}_{i}=\bigcup_{j:\left|\omega_{j}-\omega\right|<T} \mathbf{S}_{i}\left(\omega_{j}\right)$, where $U$ the merging operation and $T$ an orientation threshold. In contrast, in WIFE, we do not require from users to know their orientation and construct the RM according to two possible formats,

$$
\begin{gathered}
\mathbf{S}_{i}^{A}=\bigcup_{j=1, \ldots, 8} \mathbf{S}_{i}\left(\omega_{j}\right), \forall i \in\{1, \ldots, r\} \\
\mathbf{S}_{i}^{B}=\left[\mathbf{S}_{i}\left(\omega_{1}\right), \mathbf{S}_{i}\left(\omega_{2}\right), \ldots, \mathbf{S}_{i}\left(\omega_{8}\right)\right]^{T}, \forall i \in\{1, \ldots, r\} .
\end{gathered}
$$

In the first case the $S S$ fingerprint $\mathbf{S}_{i}^{A}$ actually merges the $S S$ fingerprints from all 8 orientations, whereas in the second case, $\mathbf{S}_{i}^{B}$, we differentiate among the $S S$ fingerprints from the 8 different orientations and consider them as 8 individual $R M$ entries, even though their physical location is identical.

\subsection{Signal Strength Filtering}

Uncontrollable environmental changes distort the SS characteristics and prevent the RF-based location systems from being accurate, (see section 3.2). We try to cope with this problem with a simple, not hardware-dependent solution. We believe that even though these environmental changes are random, their impact in the sample space can be detected and alleviated. Consider a set of SS samples (for a given point and a specific AP) most of which have absolute value higher than zero and some of them with value very close to zero. It is obvious, that since signal from this AP is received, this point is within the AP's range and the almost zero-valued samples are most probably due to the instantaneous blocking effect of obstacles temporally placed during sampling. However, considering these zero values in the average 1 reduces the correspondence with the real strength level and the higher the strength of the signal the worse this distortion is, which is even more undesirable since high SS values are more indicative and significant for the location estimation process.

${ }^{1}$ The average for summarizing the SS samples is chosen as explained in section 4.5 
Based on this observation, we propose the incorporation of a Sample Filtering module after the SS sampling step. This additional module initially removes the almost zero-valued samples in the case of existence of non-zero valued samples. More complicated filtering approaches, taking into account the frequency of the samples added complexity without an accuracy improvement, at least for our experimental data.

\subsection{Fingerprint-Based Positioning Algorithm}

During the online phase the $S S$ fingerprint of a user $u$, denoted as $\mathbf{S}_{u}$ is compared with the $R M S S$ fingerprints, $\mathbf{S}_{i}, i \in\{1, \ldots, r\}$ and the entries corresponding to the most similar ones are retrieved. The way of defining the format of an $S S$ fingerprint, the similarity between them and the way of selecting the $R M$ entries are defined by the positioning algorithm. In general, the positioning techniques are categorized into two main types: namely, deterministic and probabilistic. Since WIFE is a user-based positioning system we focus on deterministic schemes, which are simpler and have less processing requirements than the probabilistic.

In the deterministic approaches, a single scalar, usually the mean value of the measured SS samples is the format selected for representing the fingerprints. Accordingly, the merging operation in equation (1) is the average. The metric for quantifying the similarity between them is their distance in signal space, i.e.

$$
d_{u i}=\left|\mathbf{S}_{u}-\mathbf{S}_{i}\right|=\sum_{l=1}^{n}\left|S S_{u l}-S S_{i l}\right|
$$

where $n$ the total number of APs. The basic idea of the positioning algorithm is the Nearest Neighbor in Signal Space (NNSS) concept, since the closeness in signal information is considered. According to this, the RM entry $i$ with minimum $d_{u i}$ is selected as the $\mathrm{NN}$ of the user $u$. If more than one closest matches need to be determined we have the $k$-NNSS positioning algorithm, where $k$ is a parameter defining the number of these $N N \mathrm{~s}$.

We propose a modified version of the $k$-NNSS positioning algorithm which actually defines a different method for searching the NNs in the $R M$. Before giving the details of the proposed algorithm, we present the motivation behind it.

After the orientation-specific calibration, an increased number of calibrated points is available, i.e. if $r$ is the number of physically distinct points, a total number of $8 \times r S S$ fingerprints is available. Including them individually in the RM, see (2), and considering all of them during the online searching phase would on the one hand increase the probability of finding a closest match in SS but on the other hand, a large search space would degrade the time response and the resource utilization performance. Furthermore, even though orientation is an important factor for SS variations, the actual location still remains the main indicative one and thus, it should be prioritized during the SS similarity testing. Finally, the phenomenon of aliasing, as addressed in section 3.3 . would become more possible since the probability of two physically far located points having the same SS characteristics would increase. In other words, both complexity and 
accuracy related reasons triggered us in proposing a two-level algorithm, called $m k-N N S S$,

1. Initially, we consider (10) as the RM representation and the $m$ NNs are determined.

2. In the second level, we consider (2) as the RM representation but only for the selected $m N N \mathrm{~s}$, leading to a search space of $8 \times m$ points, and the $k$ NNs are finally determined.

\subsection{Location Estimation}

After having selected the $k$ NNs of user $u$, their locations are utilized for estimating the unknown location of the user. The most common method is to simply average their coordinates and take the corresponding result as the location estimate. Thus,

$$
(\widehat{x}, \widehat{y})=\left(\frac{\sum_{i \in \mathcal{N}_{u}} x_{i}}{k}, \frac{\sum_{i \in \mathcal{N}_{u}} y_{i}}{k}\right)
$$

where $\mathcal{N}_{u}$ the set of the $k$ NNs of users $u$. Different approaches can also be used, like a weighted sum of the NNs' coordinates but we prefer to maintain the positioning system as simple as possible. Furthermore, for our experiments the simple average gave the best accuracy.

\section{Enhancements}

In this section further enhancements are discussed for further improving the performance of the WIFE system.

\subsection{Number of Access Points}

An important parameter is the number of APs from which the received SS is sampled and considered in the final format of the SS fingerprint. In general, it holds that the more the available information, the more accurate the final decision. However, this statement is more rational for triangulation-based positioning techniques, according to which the position of a device is estimated as the overlapping region of the surrounding APs' ranges. In our fingerprint-based approach the similarity between fingerprints is a single scalar which contains SS information from all APs without discriminating among them (see (3) ). Thus, if many APs are considered it is more possible two different fingerprints have the same distance from the user fingerprint, and consequently fusing the system.

\subsection{Number of Nearest Neighbors}

In this section we explore the influence of the parameters $m$ and $k$, of the $m k$ NNSS algorithm, on the system performance.

Parameter $\boldsymbol{m}$. Obviously, higher values of $m$ result in larger search space at the second level of the $m k$-NNSS. Thus, the processing time and the consumed resources for performing more comparisons are increased. Its effect on the 
accuracy is not so obvious. A bigger search space increases the probability of finding a very similar point but also increases the probability of aliasing.

Parameter $\boldsymbol{k}$. Parameter $k$ defines how many NNs are finally selected for estimating the unknown location. Thus, the computational complexity is not affected by its value. However, it does affect the accuracy.

Intuitively, considering more points increases the accuracy but adding locations which are far from the real position may distort the final result. Thus, finding the optimal value is an issue. In most works which follow the $k$-NNSS method the value of $k$ is fixed for all user-cases and is more related to the grid-geometry employed during the calibration phase. However, the implicating factors of an indoor environment make each case different from the others. Therefore, making the value of $k$ adaptive for each user, i.e. $k_{u}$ instead of a fixed $k \forall u$, appears to be promising for increasing the accuracy.

The distance in SS, $d_{u i}$, would maybe give a hint for the optimal value of $k_{u}$. Assuming a fixed value for $k$, we observed that for some users, $k$ or more NNs had relatively small $d_{u i}$ but for some other users only a subset of these $k N N \mathrm{~s}$ had relatively small $d_{u i}$. Thus, adding or excluding $N N$ s based on the relativity of their $d_{u i}$ value can lead to the optimal value of $k_{u}$.

The following algorithm, AdaptiveK (Du,rlt), gives the details for estimating the optimal value of $k_{u}$ for user $u$. The input $\mathrm{Du}$ is a vector of SS distances between $u$ and the RM entries, sorted in ascending order and rlt is a parameter which defines the number of these distances that should be considered for defining a relatively small distance in SS. The operations mean and std give the mean value and the standard deviation of the first rlt smallest distances, respectively, and their purpose is to define the relatively small distance (RSdist) and the relatively small deviation (RSdev). The main idea of the algorithm is: if the absolute difference between the distance in SS of a NN $i$ and the RSdist is smaller than the RSdev, then this NN should be considered as candidate for estimating the unknown location of user $u$.

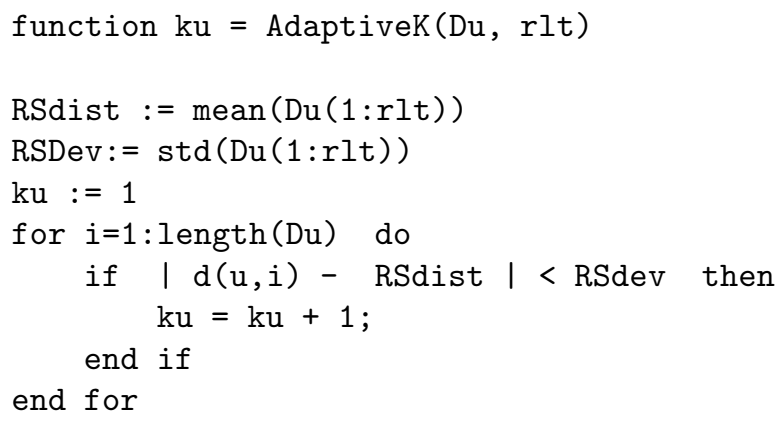

\section{Performance Evaluation}

For evaluating the performance of the WIFE system we chose to use a real experimental scenario instead of simulating one. Also, instead of performing a new 
experiment we preferred to utilize the already available measurement results from another one performed for the same purpose, [10, so that we can fairly compare our schema with other systems. In this section, we first describe the details of the experimental environment and the process for collecting the data appropriate for the positioning algorithm. After defining the performance metrics, we depict the performance improvement achieved by our proposed schema over two other positioning systems for the same experimental scenario.

\subsection{Experiment Specifications}

Test Environment. The test environment where the experiment was performed corresponds to the hallway of an office building on the campus of the University of Mannheim. The floor plan of the operation area is nearly $15 \times 36 \mathrm{~m}^{2}$.

Hardware and Software setup. As described in [4] and 10], the test environment is equipped with five Linksys/Cisco WRT54GS and four Lancom L-54g APs. All APs support 802.11b and 802.11g. One Lancom and all Linksys APs are located on the same floor with the testing area whereas three Lancom APs are located in other places inside the building.

As a client, a Lucent Orinoco Silver PCMCIA network card supporting 802.11b was used. This card was plugged into an IBM Thinkpad R51 running Linux kernel 2.6.13 and Wireless Tools 28pre. To collect signal strength samples, the implemented framework contained two parts: a library cooperating with the network card driver to perform scans and capture internal driver information, and an easy-to-use application that stores this information in a file together with additional data such as the physical position and a timestamp. Further, the application configures the library to select a scan frequency and scan technique for the signal strength measurements. For these experiments active scanning was used. Active scanning is defined in the 802.11 standard1 and it is a technique to find a suitable gateway to the Internet by measuring the SS of APs within communication range. Therefore, the available data we had and used for testing WIFE was multiple scans with the following information,

- t: timestamp in milliseconds

- id: MAC address of the scanning device

- pos: the physical coordinate of the scanning device

- degree: orientation of the user carrying the scanning device in degrees

- $\mathrm{MAC}_{i}$ : MAC address of a responding peer $i$ (e.g. an AP or a device in adhoc mode) with the corresponding values for signal strength in $\mathrm{dBm}$, the channel frequency and its mode (access point $=3$, device in adhoc mode $=1$ ).

For keeping consistency throughout the paper, we did not discriminate between the cases of whether the responding peer is an access point or a device in adhoc mode, i.e. mode 1 or mode 3. Thus, we refer to each responding peers as an access point, leading to an operational area covered in total by 17 APs (the total number of unique MAC addresses). 
To obtain the orientation of the user the Silicon Laboratories C8051F350 Digital Compass Reference Design Board was used. This device provides a USBto-Serial bridge to access the data and is powered by the USB electricity supply. The compass in the middle of the operation area was calibrated. In a closer area around the calibration point a variation of $1^{\circ}$ was measured. However, variations up to $23^{\circ}$ were rarely detected at a few points of the testing area. These measurement errors occurred always close to electro magnetic objects such as high-voltage power lines and electronic devices.

Data Collection. A grid of reference points was applied to the operation area including 166 points with a spacing of 1 meter. During the offline phase, the signal strength was measured at these reference points for different orientations. At each reference point and for each orientation 110 signal strength measurements. This led to 146,080 measurements for the offline phase. Almost 10 hours were spent to collect all the data. For the online phase 60 coordinates and orientations were randomly selected and at each one of them 110 signal strength measurements were sampled, leading to 6,600 measurements in total.

\subsection{Performance Evaluation Metrics}

A user-based positioning system is successful when it can estimate the user current location accurately, fast and without wasting the limited user-device resources. As accuracy metric we choose the Mean Location Error (MLE) between the real and estimated locations, denoted as $\left(x_{u}, y_{u}\right)$ and $\left(\widehat{x}_{u}, \widehat{y}_{u}\right)$, respectively,

$$
M L E=\sum_{u=1}^{N} \sqrt{\left(x_{u}-\widehat{x}_{u}\right)^{2}+\left(y_{u}-\widehat{y}_{u}\right)^{2}}
$$

where $N$ the total number of users. For our data $N=60$.

For evaluating the computational complexity, we measure the number of comparisons that need to be made during the online phase. For the $m k-N N S S$ this is $(166+m \times 8)$, thus it is directly related to the parameter $m$.

\subsection{Simulation Results}

In this section we evaluate the performance of WIFE for the real experiment we already described. To facilitate the comprehension of the following figures, we employ the following naming for the different cases examined,

- MergedRM is the case when orientation is not considered, i.e. only (1) is used during the Radio Map construction.

- FullRM is the case when only (2) is used.

- HierarchicalRM when both (1) and (2) are used.

In fig. 2, the superiority of the FullRM cases over the MergedRM cases illustrates the improvement in $M L E$ after orientation-specific calibration. $M L E$ is further decreased for both cases after applying the SS Sample Filtering module. 


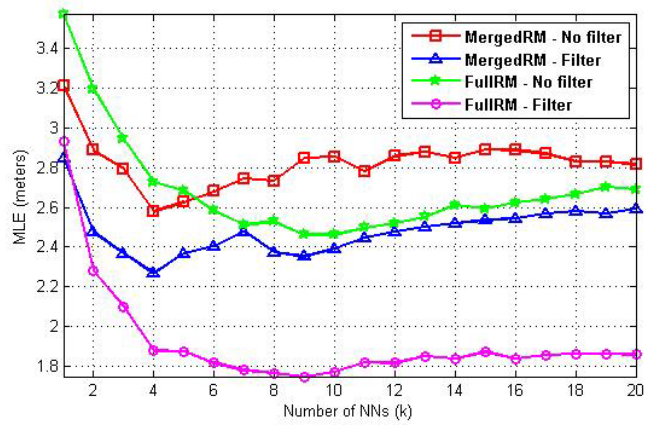

Fig. 2. Accuracy improvement after orientation-specific calibration and filtering

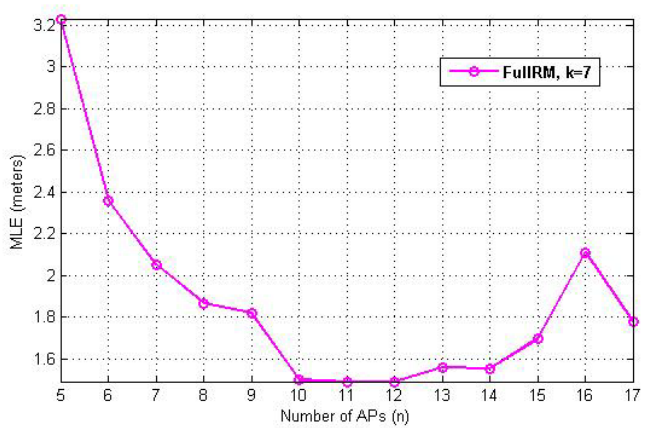

Fig. 3. MLE versus number of Access Points

The $x$ axis corresponds to the number of NNs, $k$. The best accuracy is achieved when we perform both enhancements and $k=7-10$.

In fig. 3 we examine if we can further improve this best achieved accuracy by changing the number of APs, $n$, that should be considered in (3). The $x$ axis corresponds to the number of APs. Note that it is important not only how many APs but also which APs are considered. However, drawing such conclusions was not trivial since the large number of the available APs would lead to a big number of possible combinations. Thus, we decided to first consider only the mode- 1 APs (real APs) and then include the mode-3 APs (peers in adhoc mode), since the former were more frequently scanned. We observe that the $M L E$ is improving as $n$ increases until it exceeds a certain value. This justifies our claim that more information improves the accuracy but at the same time the phenomenon of aliasing becomes more possible. The minimum $M L E$ is achieved when $n=11$ APs, which is also the average number of the visible APs from each position. Thus, a general conclusion that could be made after these observations is that, for each online user case $u$ an AP $l$ should be included in (3) if it is visible by this user $u$, i.e. $S S_{u l} \neq 0$. 


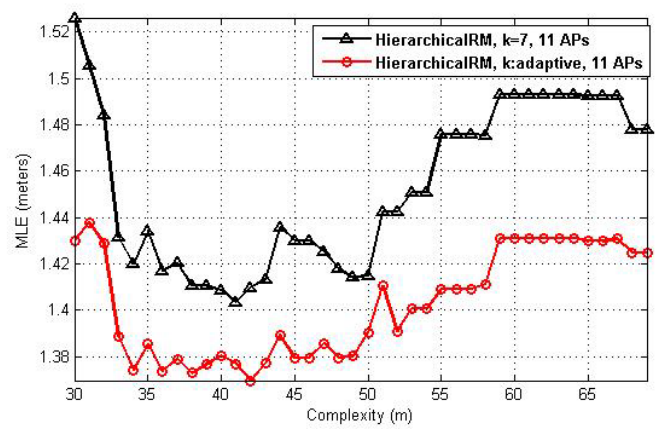

Fig. 4. MLE versus $m$ for $k=7$ or $k$ : adaptive

Table 1. Comparison with other systems

\begin{tabular}{lllll}
\hline System & $\begin{array}{l}\text { Average Error Worst Error Complexity } \\
(\text { meters })\end{array}$ & $($ meters $)$ & Type \\
& $(\sharp$ comparisons $)$ & \\
\hline WIFE- $k=7$ & 1.41 & 4.10 & $166+8 \times 42$ & Deterministic \\
WIFE- $k$ adaptive & 1.37 & 3.93 & $166+8 \times 34$ & Deterministic \\
RADAR & 2.26 & 15 & 166 & Deterministic \\
COMPASS & 1.65 & 11 & $2 \times 166$ & Probabilistic \\
\hline
\end{tabular}

The main drawback of the FullRM scheme is the large size of the resulting Radio Map, which degrades the time response and resource utilization. The hierarchicalRM scheme was proposed for reducing the time for searching the possible locations. In fig 4 the $M L E$ is shown for different values of $m$ and for the cases when $k$ is either 7 or adaptive, as described in section 5.2. We observe that the HierarchicalRM can be more accurate than the FullRM scheme and this justifies our motivation behind the $m k$-KNSS algorithm. Making $k$ adaptive improves further the system performance. Regarding the parameter $m$, we see that for $m=34-50$ the accuracy is optimal.

Finally Table 1 compares the performance of WIFE with RADAR and COMPASS for the same experiment.

\section{Conclusion - Future Venues}

In this paper we have presented WIFE, a WLAN-based system for inferring user location accurately and with low processing requirements. The main innovations proposed are: including the orientation information while training the system, performing a simple processing on the SS sample set and a two-level positioning algorithm for selecting the most probable locations. We also examined the impact on the accuracy if we consider the SS from less APs and if, for each user case, we adapt the number of calibrated points selected as possible locations. As future work, we plan to experiment the WIFE system in our own testbed. 
Acknowledgments. We would like to thank the CRAWDAD - Community Resource for Archiving Wireless Data At Dartmouth for the provision of the experimental data we used for evaluating our system.

\section{References}

1. Bahl, P., Padmanbhan, V.N.: RADAR: An In-Building RF-based User Location and Tracking System. In: IEEE Infocom 2000, vol. 2, pp. 775-784 (2000)

2. Bahl, P., Padmanbhan, V.N.: Enhancements to the RADAR User Location and Tracking System, Technical Report MSR-TR-2000-12, Microsoft Research, Microsoft Corporation One Microsoft Way Redmond, WA 98052 (February 2000)

3. Youssef, M., Agrawala, A.: The Horus Location Determination System. In: Mobisys 2005, pp. 205-219 (2005)

4. King, T., Kopf, S., Haenselmann, T., Lubberger, C., Effelsberg, W.: COMPASS: a Probabilistic Indoor Positioning System Based on 802.11 and Digital Compasses. In: WinTeck 2006, pp. 24-40 (2006)

5. Want, R., Hopper, A., Falcao, V., Gibbons, J.: The Active Badge Location System. ACM Transactions on Information Systems 1992 40(1), 91-102 (1992)

6. Priyyantha, N.B., Chakraborty, A., Balakrishnan, H.: The Cricket LocationSupport System. In: 6th International ACM MOBICOM 2000 (2000)

7. Ni, L.M., Liu, Y.: LANDMARK: Indoor Location Sensing Using Active RFID. In: Wireless Networks 2004, pp. 701-710 (2004)

8. Chen, Y.C., Chiang, J.R., Chua, H.H., Huang, P., Tsui, A.W.: Sensor Assisted WiFi Indoor Location System for Adapting to Environmental Dynamics. In: MSWiM 2005, pp. 118-125 (2005)

9. Hightower, J., Borrielo, G.: Location Systems for Ubiquitous Computing. In: IEEE 2001 (2001)

10. http://crawdad.cs.dartmouth.edu/meta.php?name=mannheim/compass 